\title{
Atomic-Scale Control of Electron Transport through Single Molecules
}

\author{
Wang, Y. F.; Kroger, J.; Berndt, R.; Vázquez, Héctor; Brandbyge, Mads; Paulsson, Magnus
}

Published in:

Physical Review Letters

Link to article, DOI:

10.1103/PhysRevLett.104.176802

Publication date:

2010

Document Version

Publisher's PDF, also known as Version of record

Link back to DTU Orbit

Citation $(A P A)$ :

Wang, Y. F., Kroger, J., Berndt, R., Vázquez, H., Brandbyge, M., \& Paulsson, M. (2010). Atomic-Scale Control of Electron Transport through Single Molecules. Physical Review Letters, 104(17), 176802.

https://doi.org/10.1103/PhysRevLett.104.176802

\section{General rights}

Copyright and moral rights for the publications made accessible in the public portal are retained by the authors and/or other copyright owners and it is a condition of accessing publications that users recognise and abide by the legal requirements associated with these rights.

- Users may download and print one copy of any publication from the public portal for the purpose of private study or research.

- You may not further distribute the material or use it for any profit-making activity or commercial gain

- You may freely distribute the URL identifying the publication in the public portal 


\title{
Atomic-Scale Control of Electron Transport through Single Molecules
}

\author{
Y.F. Wang, ${ }^{1}$ J. Kröger, ${ }^{1, *}$ R. Berndt, ${ }^{1}$ H. Vázquez, ${ }^{2}$ M. Brandbyge, ${ }^{2}$ and M. Paulsson ${ }^{3}$ \\ ${ }^{1}$ Institut für Experimentelle und Angewandte Physik, Christian-Albrechts-Universität zu Kiel, D-24098 Kiel, Germany \\ ${ }^{2}$ DTU Nanotech - Department of Micro and Nanotechnology, NanoDTU, Technical University of Denmark, \\ DK-2800 Kongens Lyngby, Denmark \\ ${ }^{3}$ School of Pure and Applied Natural Science, University of Kalmar, SE-39182 Kalmar, Sweden
}

(Received 6 February 2010; published 27 April 2010)

\begin{abstract}
Tin-phthalocyanine molecules adsorbed on $\mathrm{Ag}(111)$ were contacted with the tip of a cryogenic scanning tunneling microscope. Orders-of-magnitude variations of the single-molecule junction conductance were achieved by controllably dehydrogenating the molecule and by modifying the atomic structure of the surface electrode. Nonequilibrium Green's function calculations reproduce the trend of the conductance and visualize the current flow through the junction, which is guided through moleculeelectrode chemical bonds.
\end{abstract}

DOI: 10.1103/PhysRevLett.104.176802

The transport of electrons through molecules is relevant for processes in many branches of science and important for potential applications in electrical engineering [1-11]. While the importance of atomic structure and bonding for transport through single-molecule junctions has repeatedly been emphasized, investigations with atomic-scale control of the junction geometry are scarce. Recent scanning tunneling microscope (STM) experiments have provided structural information about the electrodes and the orientation of contacted molecules with atomic precision [12-16]. This opens an avenue for understanding and controlling the current flow through single molecules by combining transport measure ments with the atomic manipulation capabilities of a STM.

Here, we present STM measurements of the conductance of tin-phthalocyanine $(\mathrm{SnPc})$ adsorbed on a $\mathrm{Ag}(111)$ surface and contacted by a Ag-covered W tip. By systematically manipulating the chemical bonding between $\mathrm{SnPc}$ and $\mathrm{Ag}(111)$ through selective dehydrogenation of $\mathrm{SnPc}$ together with an atomic-scale structuring of the $\operatorname{Ag}(111)$ electrode, the conductance of single-molecule junctions was controllably varied from 0.013 to $0.32 G_{0}$ $\left(G_{0}=2 e^{2} / h\right.$, where $e$ is electron charge and $h$ is Planck's constant). Ab initio calculations using density functional methods and nonequilibrium Green's function techniques $[17,18]$ enable a quantitative analysis of electron transport through $\mathrm{Ag}-\mathrm{SnPc}-\mathrm{Ag}$ junctions and a visualization of the guided current flow.

Experiments were performed with a homemade STM operated at $7 \mathrm{~K}$ and in ultrahigh vacuum with a base pressure of $10^{-9} \mathrm{~Pa} . \mathrm{Ag}(111)$ and $\mathrm{W}$ tips were cleaned by $\mathrm{Ar}^{+}$bombardment and annealing. Molecules were evaporated from a heated crucible and deposited onto the sample surface at room temperature. Single $\mathrm{Ag}$ atoms, which were used to structure the surface electrode by tip manipulation, had been transferred from the tip by controlled tip-surface contacts [19].
PACS numbers: 73.63.Rt, 68.37.Ef, 68.43.Fg, 72.10.-d

The leftmost images of Figs. 1(a) and 1(b) show that SnPc exhibits two configurations upon adsorption on $\operatorname{Ag}(111)$ [20]: configurations with central protrusions (depressions) in constant-current STM images correspond to molecules with their central Sn atom pointing towards vacuum (the surface) and are referred to as SnPc-up (SnPc-down) in the following. Both SnPc configurations may be easily dehydrogenated by placing the STM tip above a ligand and applying a voltage pulse. As a result, the two peripheral $\mathrm{H}$ atoms were dissociated from the isoindole ring as reported for $\mathrm{CoPc}$ on $\mathrm{Au}(111)$ [21]. A sequence of STM images of dehydrogenated SnPc molecules together with sketches of the calculated relaxed molecule structure are presented in Figs. 1(a) and 1(b). Ligands whose outer $\mathrm{H}$ atoms have been removed appear shorter than their intact counterparts. As shown by STM images and density functional calculations, for SnPc-up and SnPc-down dehydrogenation induces a marked change of the molecular structure. After removing $\mathrm{H}$ atoms, the dehydrogenated ligand bends towards the $\mathrm{Ag}(111)$ surface and forms a chemical bond between two unsaturated $\mathrm{C}$ atoms and $\mathrm{Ag}(111)$. The smallest C-Ag separation appearing in the calculations of the relaxed binding geometry is $\approx 2 \AA$, which leads to a much stronger binding of the molecule to $\operatorname{Ag}(111)$ than in the intact configuration.

In a first step the conductance of intact and dehydrogenated molecules was measured. To this end the tip was approached toward the Sn ion at a speed of $45 \AA^{-1} \mathrm{~s}^{-1}$ and the conductance was simultaneously recorded. Figure 2(a) presents experimental results for SnPc-up and its dehydrogenated products. The displacement axis shows the tip excursion towards the molecule with zero displacement corresponding to the position of the tip before opening the feedback loop of the microscope. The conductance increases exponentially in the tunneling region and corresponds to an apparent barrier height of $4.3 \mathrm{eV}$, which is slightly larger than the work function of clean $\mathrm{Ag}(111)$. At a conductance of $\approx 0.005 G_{0}$, the slope of the conductance 

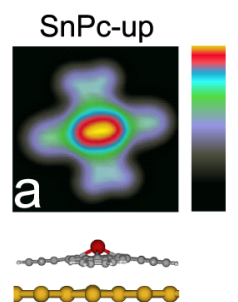

$\mathrm{G}=0.052 \mathrm{G}$
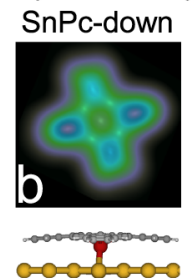

$\mathrm{G}_{\mathrm{c}}=0.32 \mathrm{G}_{0}$

AgSnPc-up
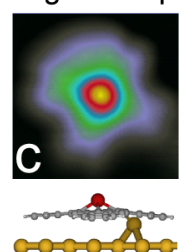

$\mathrm{G}_{\mathrm{c}}=0.058 \mathrm{G}_{0}$
$-2 \mathrm{H}$
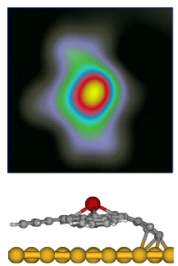

$0.068 \mathrm{G}_{0}$

$-2 \mathrm{H}$
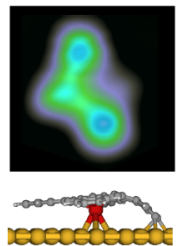

$0.24 \mathrm{G}_{0}$
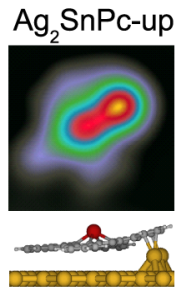

$0.025 \mathrm{G}$
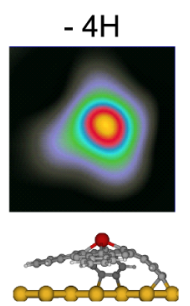

$0.020 \mathrm{G}$

$-4 \mathrm{H}$
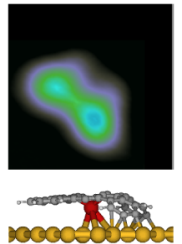

$0.30 \mathrm{G}_{0}$
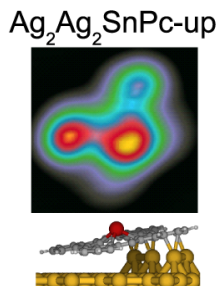

$0.030 \mathrm{G}_{0}$
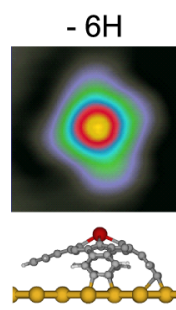

$0.018 \mathrm{G}$ $-6 \mathrm{H}$

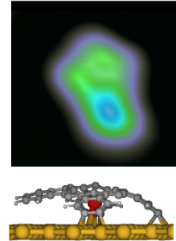

$0.28 \mathrm{G}$
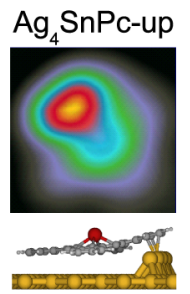

$0.019 \mathrm{G}_{0}$
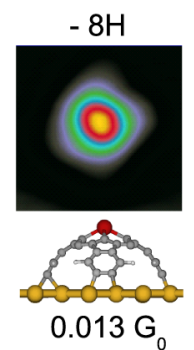

$-8 \mathrm{H}$

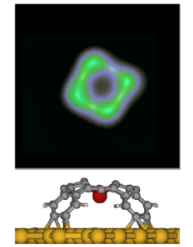

$0.08 \mathrm{G}_{0}$

$\mathrm{Ag}_{4} \mathrm{SnPc}$-down

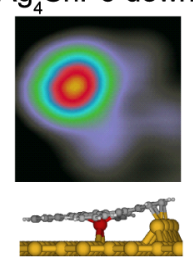

$0.054 \mathrm{G}_{0}$
FIG. 1 (color online). Constant-current STM images $(17 \AA \times 17 \AA, 80$ pA, voltages between -0.2 and $0.2 \mathrm{~V}$ ) of (a), (b) SnPc-up and SnPc-down in their intact and progressively dehydrogenated $(-n \mathrm{H}, n=2,4,6,8)$ configurations and of (c) intact $\mathrm{SnPc}$ molecules supported by Ag clusters of various sizes. The color scale ranges from black (low) to light yellow (high). Apparent heights depend on the applied voltage. Calculated adsorption structures and experimental contact conductances, $G_{c}$, are indicated below each image. trace increases abruptly, which signals the transition to the contact region. The contact conductance $G_{c}$ is defined by the intersection of linear fits to the transition and the contact regions as illustrated by the solid lines in Fig. 2(a) and introduced in Refs. [14,15]. For SnPc-up a contact conductance of $\approx 0.052 G_{0}$ is obtained in this way. SnPc-up molecules, from which two $\mathrm{H}$ atoms were removed (SnPc-up-2H), exhibit similar conductance curves, albeit with a slightly higher contact conductance of $\approx 0.068 G_{0}$. Starting from SnPc-up- $4 \mathrm{H}$, the transition from tunneling to contact occurs with an appreciably lower slope and the contact conductances become smaller with increasing degree of dehydrogenation (SnPc-up-4H, $0.020 G_{0}$; SnPc-up-6H, 0.018G $G_{0}$; SnPc-up-8H, 0.013G $G_{0}$. A similar trend of the conductance evolution with increasing number of removed $\mathrm{H}$ atoms is observed for SnPcdown [Fig. 2(b)]. However, the conductances are considerably higher than for SnPc-up.

To understand the trends of conductance evolution with dehydrogenation, first-principles simulations (SIESTA [18]) and subsequent transport calculations (TRANSIESTA [17]) were performed. The unit cell for transport calculations consists of 509 atoms comprising 56 atoms per $\mathrm{Ag}$ layer (4 layers for the substrate and for the tip), 4 atoms for the pyramidal tip, and 57 atoms for the intact molecule. The geometry was optimized by minimizing the molecular and tip forces in a smaller cell having two or three Ag layers. The calculations model the experimental findings in a qualitative way. Deviations are most likely due to van der Waals forces, which influence the junction geometry and are not properly accounted for in density functional theory. Calculated contact conductances were therefore evaluated at fixed tip-Sn distances $(3.0 \AA$ for SnPcup- $n \mathrm{H}, 4.0 \AA$ for Sn-Pc-down- $n \mathrm{H}$ junctions), which were extracted from simulated conductance curves showing a clear transition between tunneling and contact. While the calculated conductances scale with the tip-Sn distance, the trends depicted in Figs. 2(c) and 2(d) are not affected significantly.

Individual transport channels were calculated to find the contributions of the $\mathrm{Sn}$ atom, the $\pi$ molecular orbitals, and the $\mathrm{C}-\mathrm{Ag}$ chemical bonds between the (dehydrogenated) molecule and $\operatorname{Ag}(111)$ to the total conductance at contact [22]. For the SnPc-up series, the transmission is dominated by the $\pi$ orbitals, which on $\mathrm{Ag}(111)$ are located slightly above the Fermi level $[\approx 0.08 \mathrm{eV}$, Fig. 2(c)], and which originate from the degenerate lowest unoccupied molecular orbital [23]. Upon dehydrogenation C-Ag chemical bonds are formed, which leads to a bending of the molecule and a concomitant lifting of the central Sn ion from the surface. Together with the mechanical distortion of the molecule, the modification of its $\pi$ electronic system gives rise to a shift of the transmission peak away from the Fermi level [Fig. 2(c)], which in turn leads to a decrease of contact conductances of the dehydrogenated SnPc products. In particular, SnPc-up-8H exhibits a highly distorted molecular plane with Sn lifted away from the surface by $1.0 \AA$ compared to the intact molecule. This molecule further shows the lowest contact conductance. The stronger chemical bonding of dehydrogenated molecules may explain the smooth transition to contact observed from SnPcup- $4 \mathrm{H},-6 \mathrm{H},-8 \mathrm{H}$ since the resulting relaxations of the 

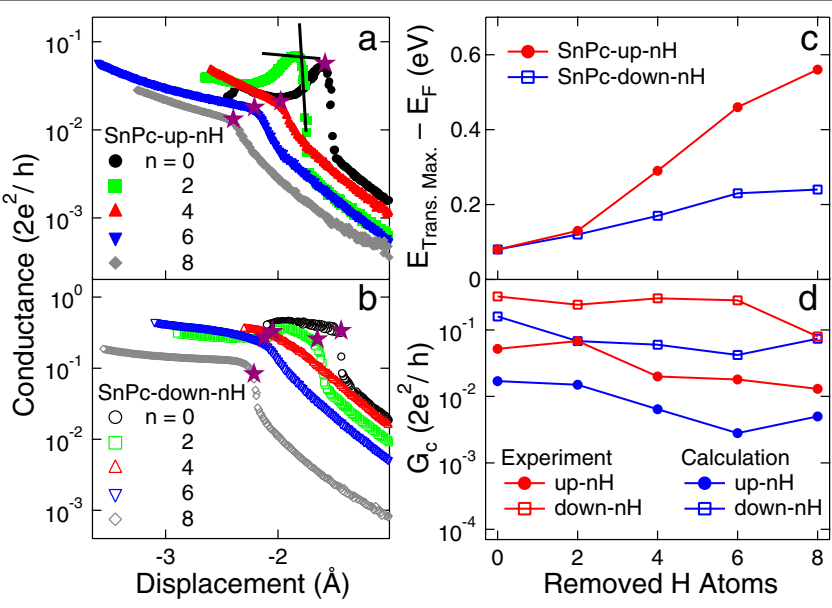

FIG. 2 (color online). (a) Conductance versus tip displacement of SnPc-up- $n \mathrm{H}(n=0,2,4,6,8)$. The conductance curves have been horizontally shifted for clarity $(2 \mathrm{H},-0.8 \AA ; 4 \mathrm{H},-0.2 \AA$; $6 \mathrm{H}, 0.4 \AA ; 8 \mathrm{H},-0.4 \AA$ ). (b) Like (a) for SnPc-down- $n \mathrm{H}(2 \mathrm{H}$, $-0.2 \AA ; 4 \mathrm{H},-0.5 \AA ; 6 \mathrm{H}, 0.8 \AA ; 8 \mathrm{H},-0.8 \AA$ ). Zero displacement is defined by feedback loop parameters of $0.1 \mathrm{~V}, 10 \mathrm{nA}$. Solid lines in (a) are linear fits to the transition region and a part of the contact region. The intersection of these fits defines the contact conductance, which is indicated by stars for all other curves. (c) Calculated energy of the transmission maximum as a function of $n$ (Fermi energy is set to zero). (d) Comparison of experimental (red) and calculated (blue) contact conductances $\left(G_{c}\right)$ of SnPc-down (squares) and SnPc-up (circles).

molecule are reduced. SnPc-up and SnPc-up-2H, which are more weakly bonded to the surface, exhibit a rather abrupt tunneling-to-contact transition. A similar behavior was reported for adsorbed $\mathrm{Ag}$ and $\mathrm{Cu}$ atoms [19]. The different chemical bondings may also explain the local maxima observed in conductance curves of SnPc-up- $n \mathrm{H}(n=$ $0,2)$ and of SnPc-down-2H. Since again van der Waals forces may play an important role in the junction relaxations the calculations are unable to mimic this effect.

The conductance at contact of the SnPc-down series is also determined by the $\pi$ orbitals, with an additional contribution of the $\mathrm{Sn}$ atom owing to its binding to $\mathrm{Ag}(111)$. The most conducting channel is responsible for $\approx 70 \%$ of the total transmission, while this figure is $\approx 30 \%$ for the next most conducting channel. Both channels show $\pi$ character and have a significant weight on the Sn atom. Consequently, SnPc-down exhibits a higher conductance than SnPc-up. Similar to the SnPc-up series, progressive dehydrogenation gives rise to a continued decrease of contact conductance. For SnPc-down-8H the strong molecular deformation lifts the Sn atom by $0.7 \AA$ from the $\operatorname{Ag}(111)$ surface compared to the intact molecule and its coupling to the substrate is efficiently reduced.

These results show that by modifying the chemical bonding between molecule and surface the conductance of the molecular junction may be tuned in a controlled way. To obtain the current pathway from molecule to surface, all local currents flowing in the bonds between individual

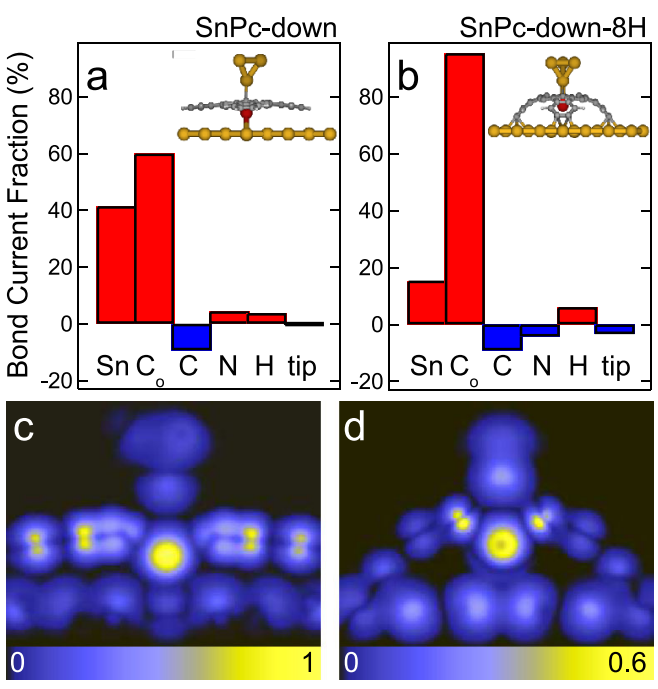

FIG. 3 (color online). (a),(b) Bond current fractions for SnPcdown and SnPc-down-8H originating from bonds between $\operatorname{Ag}(111)$ and $\mathrm{Sn}, \mathrm{C}$ atoms at the end of the ligands $\left(\mathrm{C}_{o}\right)$, all other $\mathrm{C}$ atoms, $\mathrm{N}$ and $\mathrm{H}$ atoms, and the tip. (c),(d) Dominating transmission channels of SnPc-down and SnPc-down-8H. The color scale shows the amplitude of the scattering state in a cross section through the center of the molecule.

atoms in the molecule and all atoms in the surface are summed up [24-26]. Since almost the entire current flow from tip to surface is via the molecule, these individual currents for each atom in the molecule add up to the total current. Therefore, the contribution to the total current of each atom or group of atoms in the molecule and the $\operatorname{Ag}(111)$ surface was evaluated and is shown in the bar charts in Figs. 3(a) and 3(b) for SnPc-down and SnPcdown-8H. First, $\approx 60 \%$ of the total current between molecule and surface flow through the outermost $\mathrm{C}$ atoms $\left(\mathrm{C}_{o}\right)$ for the intact molecule, while $\approx 97 \%$ of the total current flow between these directly surface-bonded $\mathrm{C}$ atoms for the dehydrogenated molecule. Thus, the outermost $\mathrm{C}$ atoms of the molecule appear to play a particularly important role in electron transport through the molecule. Second, the current in the bonds between the central Sn atom and the surface contributes $\approx 43 \%$ for the intact SnPc-down molecule, while it is reduced to $\approx 16 \%$ for SnPc-down- $8 \mathrm{H}$. In other words, electron transport through a channel containing the $\mathrm{Sn}$ atom is strongly reduced upon removing the outermost $\mathrm{H}$ atoms. The current pathway through other chemical bonds between $\mathrm{C}, \mathrm{N}, \mathrm{H}$ atoms and the surface are seen to be of minor importance. Negative bond currents are directed opposite to the total current and reflect quantum interferences, which can lead to loop currents [26].

Based on the above analysis, a decrease of the conductance at contact is expected for SnPc-up and SnPc-down upon weakening the $\pi$ coupling between the molecules and the surface. This behavior is reflected in the nature of the most transmitting channel of SnPc-down showing clear $\pi$ coupling [Fig. 3(c)] in contrast to SnPc-down-8H [Fig. 3(d)], where considerable transmission is observed 
at the $\mathrm{C}-\mathrm{Ag}$ bonds [27]. To test this hypothesis further, the flat adsorption geometry of $\mathrm{SnPc}$ on $\mathrm{Ag}(111)$ was modified by moving the molecule onto Ag clusters of different sizes, which had been fabricated by atom manipulation before. As shown by STM images and sketches of the relaxed adsorption geometries [Fig. 1(c)], this atomic-scale structuring of the $\operatorname{Ag}(111)$ electrode causes a partial lifting of SnPc molecules from the $\operatorname{Ag}(111)$ surface. The degree of detaching the molecule from the surface may be controlled to some extent by the choice of the cluster size and by its position below the molecule plane. A SnPc-up molecule is weakly lifted from the $\operatorname{Ag}(111)$ surface when a single $\mathrm{Ag}$ atom is located between two adjacent ligands close to one $\mathrm{N}$ atom [Fig. 2(c), AgSnPc-up] and its contact conductance, $\approx 0.058 G_{0}$, is slightly modified compared to the flat adsorption [Fig. 1(a)]. A $\mathrm{Ag}_{2}$ cluster positioned below a ligand of SnPc-up $\left(\mathrm{Ag}_{2} \mathrm{SnPc}\right.$-up), two $\mathrm{Ag}_{2}$ clusters below two neighboring ligands $\left(\mathrm{Ag}_{2} \mathrm{Ag}_{2}\right.$ SnPc-up), and a $\mathrm{Ag}_{4}$ cluster below a single ligand $\left(\mathrm{Ag}_{4} \mathrm{SnPc}\right.$-up) progressively detach the molecule from the surface. A SnPc-up molecule supported by a $\mathrm{Ag}$ dimer or a $\mathrm{Ag}$ tetramer exhibits contact conductances of $0.025 G_{0}$ and $0.019 G_{0}$, which are lower by $50 \%$ and $70 \%$ compared to the values obtained for the flat molecule. The contact conductance slightly increased to $0.030 G_{0}$ for $\mathrm{Ag}_{2} \mathrm{Ag}_{2} \mathrm{SnPc}$-up, which may originate from an increased contact area between the molecule and the electrode. For $\mathrm{Ag}_{4} \mathrm{SnPc}$-down, the contact conductance drops by $80 \%$ to $0.054 G_{0}$ compared to $\mathrm{SnPc}$-down because of the decreased $\pi$-surface and Sn-surface coupling.

In summary, pathways of the electron current through a molecular junction were investigated using STM-based transport and manipulation experiments along with calculations. Control of the conductance and guiding of the electron current were achieved by modifying the chemical interaction of the molecule with the electrodes and by atomic-scale structuring the electrodes. This approach may be extended to electron transfer processes for a wide range of molecular junctions.

We thank A.-P. Jauho (Technical University of Denmark) for discussions, the Innovationsfonds Schleswig-Holstein, the Deutsche Forschungsgemeinschaft (SFB 677), and the Danish Research Council (FTP Grant No. 65212) for financial support. The Danish Center for Scientific Computing and the Barcelona Supercomputing Center are acknowledged for computer resources.

*kroeger@physik.uni-kiel.de

[1] M. A. Reed, C. Zhou, C. J. Muller, T. P. Burgin, and J. M. Tour, Science 278, 252 (1997).

[2] X. D. Cui, A. Primak, X. Zarate, J. Tomfohr, O. F. Sankey, A. L. Moore, T. A. Moore, D. Gust, G. Harris, and S. M. Lindsay, Science 294, 571 (2001).
[3] R. H. M. Smit, Y. Noat, C. Untiedt, N. D. Lang, M. C. van Hemert, and J. M. van Ruitenbeek, Nature (London) 419, 906 (2002)

[4] G. V. Nazin, X.H. Qiu, and W. Ho, Science 302, 77 (2003).

[5] T. Dadosh, Y. Gordin, R. Krahne, I. Khivrich, D. Mahalu, V. Frydman, J. Sperling, A. Yacoby, and I. Bar-Joseph, Nature (London) 436, 677 (2005).

[6] W. Haiss, C. Wang, I. Grace, A. S. Batsanov, D. J. Schiffrin, S. J. Higgins, M. R. Bryce, C. J. Lambert, and R. J. Nichols, Nature Mater. 5, 995 (2006).

[7] J. Repp, G. Meyer, S. Paavilainen, F. E. Olsson, and M. Persson, Science 312, 1196 (2006).

[8] L. Venkataraman, J.E. Klare, C. Nuckolls, M. S. Hybertsen, and M. L. Steigerwald, Nature (London) 442, 904 (2006).

[9] E. Lörtscher, H. B. Weber, and H. Riel, Phys. Rev. Lett. 98, 176807 (2007).

[10] F. Chen, Z. Huang, and N. Tao, Appl. Phys. Lett. 91, 162106 (2007).

[11] M. Galperin, M. A. Ratner, A. Nitzan, and A. Troisi, Science 319, 1056 (2008).

[12] J. Kröger, N. Néel, and L. Limot, J. Phys. Condens. Matter 20, 223001 (2008).

[13] L. Lafferentz, F. Ample, H. Yu, S. Hecht, C. Joachim, and L. Grill, Science 323, 1193 (2009).

[14] N. Néel, J. Kröger, L. Limot, and R. Berndt, Nano Lett. 8, 1291 (2008).

[15] N. Néel, J. Kröger, L. Limot, T. Frederiksen, M. Brandbyge, and R. Berndt, Phys. Rev. Lett. 98, 065502 (2007).

[16] R. Temirov, A. Lassise, F. B. Anders, and F. S. Tautz, Nanotechnology 19, 065401 (2008).

[17] M. Brandbyge, J.-L. Mozos, P. Ordejón, J. Taylor, and K. Stokbro, Phys. Rev. B 65, 165401 (2002).

[18] J. M. Soler, E. Artacho, J. D. Gale, A. Garía, J. Junquera, P. Ordejón, and D. Sánchez-Portal, J. Phys. Condens. Matter 14, 2745 (2002).

[19] L. Limot, J. Kröger, R. Berndt, A. Garcia-Lekue, and W. A. Hofer, Phys. Rev. Lett. 94, 126102 (2005).

[20] Y. F. Wang, J. Kröger, R. Berndt, and W. A. Hofer, Angew. Chem., Int. Ed. 48, 1261 (2009).

[21] A. Zhao, Q. Li, L. Chen, H. Xiang, W. Wang, S. Pan, B. Wang, X. Xiao, J. Yang, J. G. Hou, and Q. Zhu, Science 309, 1542 (2005).

[22] M. Paulsson and M. Brandbyge, Phys. Rev. B 76, 115117 (2007).

[23] C. Stadler, S. Hansen, I. Kröger, C. Kumpf, and E. Umbach, Nature Phys. 5, 153 (2009).

[24] T. N. Todorov, Philos. Mag. B 79, 1577 (1999).

[25] S. Nakanishi and M. Tsukada, Phys. Rev. Lett. 87, 126801 (2001).

[26] G. S. Solomon, C. Herrmann, T. Hansen, V. Mujica, and M. A. Ratner, Nature Chem. 2, 223 (2010).

[27] See supplementary material at http://link.aps.org/ supplemental/10.1103/PhysRevLett.104.176802 for a visualization of the calculated distribution of dominating transmission channels through SnPc-down and SnPcdown-8H. 